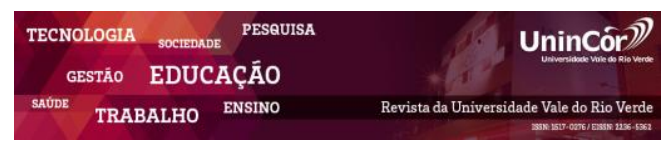

Revista da Universidade Vale do Rio Verde ISSN: 1517-0276 / EISSN: 2236-5362 v. $16 \mid$ n. $1 \mid$ Ano 2018

Rafaela Maiara Caetano Doutoranda em Administração, UFLA rafa.caet.94@gmail.com

Sheldon William Silva

Doutorando em Administração, UFLA sheldonwilliamsilva@gmail.com

Dany Flavio Tonelli

Docente do Departamento de Administração e Economia da UFLA danytonelli@dae.ufla.br

Cristina Lelis Leal Calegario Docente do Departamento de Administração e Economia da UFLA ccalegario@dae.ufla.br

Gideon Carvalho de Benedicto Docente do Departamento de Administração e Economia da UFLA gideon.benedicto@dae.ufla.br

\section{POLÍTICA INDUSTRIAL, INVESTIMENTO DIRETO EXTERNO E OS TRANSBORDAMENTOS DE PRODUTIVIDADE: UMA ANÁLISE BIBLIOMÉTRICA}

\section{RESUMO}

Uma pesquisa recente sobre política industrial mostrou que nos últimos cinco anos, pelos menos 84 países - tanto desenvolvidos quanto em desenvolvimento, adotaram estratégias formais de desenvolvimento industrial, sugerindo que as políticas industriais se tornaram presentes na conjuntura econômica mundial atual (UNCTAD, 2018). O objetivo deste artigo consiste na revisão bibliométrica dos estudos que contemplam esta temática, fazendo as devidas conexões com os principais autores ligados a esta linha de pesquisa, bem como redes de coautoria e colaboração, redes de colaboração entre países. Como método de pesquisa apresenta-se uma análise bibliométrica com a finalidade de identificar uma amostra da produção científica sobre o tema em questão. Os resultados encontrados demonstram que a rede de autores e coautores encontrada está de acordo com o referencial teórico apresentado, bem como as formulações teóricas principais. Um ponto a se destacar é que mesmo em se tratando de pesquisas cujo campo de aplicação se dá em países em desenvolvimento, a rede de autores e países (com exceção da China) ainda se concentra nos países ricos.

Palavras-chave: Política Industrial. Investimento Direto Estrangeiro. Países em Desenvolvimento. Transbordamento. Economia

\section{INDUSTRIAL POLICY, FOREIGN DIRECT INVESTMENT AND SPILLOVER PRODUCTIVITY: A BIBLIOMETRIC ANALYSIS}

\begin{abstract}
Recent research on industrial policy has shown that in the last five years at least 84 countries - both developed and developing - have adopted formal industrial development strategies, suggesting that industrial policies have become present in today's global economic environment (UNCTAD, 2018). The purpose of this article is to bibliometric review of the studies that contemplate this subject, making the necessary connections with the main authors related to this line of research, as well as co-authorship and collaboration networks, collaboration networks between countries. As a research method, a bibliometric analysis is presented with the purpose of identifying a sample of the scientific production on the subject in question. The results show that the network of authors and coauthors found is in agreement with the theoretical reference presented, as well as the main theoretical formulations. One point to note is that even in the case of research whose field of application is in developing countries, the network
\end{abstract}


of authors and countries (with the exception of China) is still concentrated in rich countries.

Keywords: Industrial Policy. Foreign Direct Investment. Developing countries. Spillover. Economy.

Recebido em: 05/09/2018 - Aprovado em: 09/12/2018 - Disponibilizado em: 15/12/2018

\section{INTRODUÇÃO}

Uma pesquisa recente sobre política industrial mostrou que, nos últimos cinco anos, pelos menos 84 países - tanto desenvolvidos quanto em desenvolvimento, representando cerca de $90 \%$ do Produto Interno Bruto (PIB) mundial - adotaram estratégias formais de desenvolvimento industrial, sugerindo que as políticas industriais se tornaram presentes na conjuntura econômica mundial atual (UNCTAD, 2018). A questão da adoção de políticas industriais é um tema bastante controverso na literatura econômica, cujo debate se constitui desde a sua aplicação, seus efeitos e duração até as suas formas de atuação.

Diante desta perspectiva, a política industrial pode ser vista como uma forma de promoção de desenvolvimento de setores econômicos fundamentais na difusão de tecnologias, geração de empregos e divisas, aumentando assim a competitividade industrial (CORONEL et al, 2014). Ademais, a política industrial refere-se às maneiras pelas quais os governos visam alcançar ou acelerar mudanças estruturais. Assim, como a maioria dos países implementa hoje alguma forma de política industrial, a questão não é mais se há um argumento a favor da política industrial, mas como ela deve ser conduzida (UNIDO, 2012).
Neste sentido, evidencia-se na literatura que a maioria dos países que hoje são considerados desenvolvidos, fizeram uso extensivo de políticas industriais para alavancar os setores considerados fundamentais. Chang (2002) afirma que os países desenvolvidos mudaram sua postura ao chegarem neste patamar de desenvolvimento, criando mecanismos para os países em desenvolvimento não utilizem das políticas industriais, "chutando a escada" que os mais ricos utilizaram para alcançar o crescimento econômico.

Associado a este cenário e tendo em vista o crescimento expressivo do investimento direto estrangeiro (IDE) em países de economias em desenvolvimento nas últimas décadas, tem-se verificado um número cada vez maior de tratados bilaterais de investimentos e acordos regionais haja vista que esta maior integração tem resultado em maiores incentivos ao investimento oferecidos pelos governos dos países hospedeiros (BANGA, 2003). Dessa forma, as políticas industriais também podem promover ligações econômicas muito necessárias de empresas locais que se tornaram importantes atores internacionais. Além disso, as atuais políticas industriais tendem a se concentrar na internacionalização, incluindo exportação e atração de IDE, bem como na atualização e aprendizado tecnológico (desenvolvimento de 
capacidades) como requisitos importantes para melhorar a competitividade dos setores de manufatura e serviços (DEVLIN \& MOGUILLANSKY, 2012). Assim, a presença desses investimentos estrangeiros em economias locais pode propiciar ganhos de produtividade por meio dos transbordamentos via transações não mercantis quando o conhecimento é dissipado para as empresas de propriedade local (BUCKLEY, CLEGG, \& WANG, 2010).

O objetivo deste artigo consiste na revisão bibliométrica dos estudos que contemplem a temática da Política Industrial, Investimento Direto Externo e Transbordamento de Produtividade, fazendo as devidas conexões com os principais autores ligados a esta linha de pesquisa, bem como redes de coautoria e colaboração, e redes de colaboração entre países.

O trabalho é relevante devido ao fato de que a revisão bibliométrica permite $\mathrm{o}$ acesso de diversos pesquisadores e interessados no tema explorado e contexto da produção científica e redes de parceria e coautoria. Para tanto, o artigo está estruturado em três sessões além desta introdução. $\mathrm{Na}$ primeira delas é colocado o referencial teórico a respeito da abordagem a ser investigada. Na segunda são feitas as considerações metodológicas a despeito da revisão bibliométrica e, por último, a terceira sessão encerra com as considerações finais.

\section{REFERENCIAL TEÓRICO}

Nessa sessão apresentam-se o marco teórico que sustenta o referido estudo. Diante dessa proposição, faz-se necessário percorrer a literatura referente a política industrial, com seus contextos e trajetória, bem como lançar seu olhar para os efeitos de transbordamento de produtividade relacionados ao investimento estrangeiro direto.

\subsection{Trajetória da Política Industrial}

A globalização mudou as realidades econômicas. A ideia de que as competências das empresas multinacionais (EMNs) estão se tornando cada vez mais móveis e intensivas em conhecimento ajudaram a mudar os conjuntos de oportunidades tanto dessas empresas quanto dos países anfitriões. As estratégias de desenvolvimento baseadas no investimento direto estrangeiro (IDE) são agora comuns entre os países menos desenvolvidos, mas há também uma maior concorrência pelos tipos certos de investimento. Em geral, o equilíbrio no poder de barganha mudou em favor da empresa multinacional, e os países menos desenvolvidos precisam cada vez mais fornecer ativos exclusivos e não replicáveis para manter uma estratégia de desenvolvimento bem-sucedida de IDE (NARULA E DUNNING, 2010).

As ligações entre o IDE e a política industrial durante os anos 1950-1970 eram complexas. As portas para entrar no mercado nacional estavam abertas a multinacionais estrangeiras, mas sob estrito controle. Esse controle reflete a natureza conflitante ainda existente entre os interesses dos governos dos países de acolhimento e das multinacionais estrangeiras: o primeiro visa alcançar os objetivos de desenvolvimento de seus países e o segundo, guiado por preocupações de mercado e lucro (DUNNING, 1998; STOPFORD, 1994; 
VERNON, 1998; RUGMAN e VERBEKE, 2001; COSTA e FILIPPOV, 2008).

Em geral, há um acordo de que a política industrial (PI) envolve formas de intervenções governamentais que tentam promover investimentos produtivos de uma forma que não ocorreria em interações de mercado livres de tais intervenções (PACK e SAGGI, 2006; CIMOLI et al., 2009;). Lazzarini (2015) argumenta que alguns autores chegam ao ponto de afirmar que é preferível arcar com uma política industrial imperfeita do que fracassar no lado oposto da inação governamental. Portanto, o papel da política industrial é fornecer incentivos para as partes interessadas do mercado explorarem a adoção de novos processos e atividades de uma ordem mais elevada, muitas vezes diante de obstáculos que não são facilmente superados pelas forças autônomas do mercado (PRIMI e PEREZ NÚÑEZ, 2009).

Ainda neste sentido, Rodrik (2010) argumenta que as forças de mercado e o empreendedorismo privado estariam no centro da agenda de desenvolvimento, mas os governos também desempenhariam um papel estratégico e coordenador na esfera produtiva, além de simplesmente garantir os direitos de propriedade, a execução de contratos e a estabilidade macroeconômica. Suzigan e Furtado (2010) também concordam que a coordenação das ações governamentais em articulação com o setor privado (este sendo protagonista) é uma questão central para a gestão da política industrial e tecnológica. Ademais, eles acreditam que a definição da instituição coordenadora seja a tarefa mais complexa ao se formular qualquer tipo de política industrial, seja no tipo da instituição, nos poderes a ela atribuídos e quais mecanismos de articulação com o setor privado serão utilizados.

Coronel et al (2014), numa discussão historiográfica sobre a política industrial, apresentam argumentos a favor de que várias nações fizeram uso de políticas industriais, especialmente na Europa, Estados Unidos e países asiáticos, apesar das controvérsias que cercam a utilização de tal política. Desde a dinastia Tudor, a Inglaterra no século XVI, utilizou-se de restrições às importações para proteger o setor manufatureiro. Esta política só foi abandonada quando sua liderança econômica já era incontestável, no século XIX. Chang (2002) acredita que a importância dada ao protecionismo norte-americano foi fundamental para o desenvolvimento do seu setor industrial. Coronel et al (2014) ainda colocam que logo após a Independência dos Estados Unidos, e a consequente concentração do desenvolvimento em duas regiões: a Sul, escravocrata; e a norte, da qual se restringiu no setor industrial e defendia proteção ao setor por meio de imposição de cotas, tarifas e sanções, bem como restrição voluntária às importações.

No que se refere aos países em desenvolvimento, os estágios iniciais da industrialização foram acompanhados pelas políticas tradicionais de industrialização por substituição de importações (ISI). Assim, a partir do Plano de Metas, o governo brasileiro promoveu o estabelecimento de indústrias intensivas em capital desde a década de 1950, incluindo as indústrias automobilística e química, por meio de subsídios e mercados domésticos protegidos. Em 1974, o país adotou 
um ambicioso programa de desenvolvimento industrial, o Plano Nacional de Desenvolvimento II (PND-II), no qual a proteção comercial foi combinada com a promoção de investimentos (nacionais e estrangeiros), com subsídios às exportações de manufaturados (DEVLIN e MOGUILLANSKY, 2012).

De um lado, tem-se que a substituição de importações, o planejamento e a propriedade estatal produziram alguns êxitos, mas onde se estagnaram com o tempo, levaram a falhas e crises colossais. Por outro, a liberalização econômica e a abertura beneficiaram as atividades de exportação, os interesses financeiros e os trabalhadores qualificados, mas, na maioria das vezes, resultaram em taxas de crescimento em toda a economia (em trabalho e produtividade total dos fatores) muito aquém daquelas experimentadas sob as políticas ruins e antigas do passado (RODRIK, 2010). Na mesma linha, Devlin e Moguillansky (2012) acreditam que as privatizações da era do Consenso de Washington contribuíram para a ampliação e diversificação das atividades econômicas dos grupos econômicos locais, algumas das quais que se tornaram multinacionais em suas atividades econômicas. No entanto, embora essas empresas tenham escala, talento e importem as mais recentes máquinas e equipamentos, muitas não têm sido líderes em desenvolvimento local ou inovação e investimento em $\mathrm{P} \& \mathrm{D}$, que é muito baixa em quase todos os países latinoamericanos.

Quanto ao relacionamento entre o IDE, reestruturação industrial e inovação, Costa e Fillippov (2008) argumentam que os anos 90 representaram um ponto de virada nas abordagens dos governos anfitriões em relação ao IDE, dado por uma combinação de vários desenvolvimentos. Primeiro, este período foi marcado por um aumento dramático nos fluxos globais de IDE. Os investimentos diretos de multinacionais estrangeiras têm sido um elemento importante no processo de reestruturação que ocorre nos setores industriais em todo o mundo, particularmente nos países em desenvolvimento (já) industrializados e naqueles de economias em transição. Um segundo grupo importante de desenvolvimentos observado na década de 1990 é a desregulação e liberalização generalizadas das economias nacionais, alinhadas com as mudanças no contexto institucional internacional, especialmente os acordos da Organização Mundial do Comércio (OMC) sobre medidas de investimento relacionadas ao comércio (TRIM), e uma infinidade de acordos bilaterais de investimento (COSTA e FILIPPOV, 2008).

2.2 Efeitos de Transbordamento sobre a Produtividade

Existem vários fatores determinantes relacionados à atração de atividades de pesquisa e desenvolvimento (P\&D) - infraestrutura física e de ciência e tecnologia (C\&T) adequada, incentivos fiscais, oferta de mão de obra qualificada, direitos de propriedade intelectual, estabilidade econômica, entre outros (ZANNATA e QUEIROZ, 2007). Tanto os formuladores de políticas quanto os acadêmicos há muito tempo sustentam a opinião de que existem "transbordamentos" do investimento direto externo (IDE), o qual pode gerar alguma forma de conhecimento indireto ou transferência 
de tecnologia da empresa controladora para a afiliada e, subsequentemente, da afiliada para a economia local (NARULA e DRIFFIELD, 2012).

Buckley, Clegg e Wang (2010) alegam que os efeitos de transbordamento podem ser refletidos na melhoria da produtividade e outros benefícios em empresas de propriedades locais. Ademais, um dos motivos pelos quais os governos interveem com políticas governamentais está baseado na crença de que essas empresas de propriedade local podem se beneficiar das empresas estrangeiras por meio de transbordamentos de produtividade (GÖRG e GREENAWAY, 2004). De acordo com Caves (1974), podem haver efeitos benéficos das empresas estrangeiras em termos de transbordamentos e que podem ser resumidos em termos de: (a) ganhos de eficiência alocativa; (b) melhorias de eficiência técnica por meio da utilização de práticas superiores; e (c) transferência de tecnologia quando a presença de empresas estrangeiras propicia empresas locais acesso à tecnologia avançada.

É importante ressaltar que o relacionamento entre IDE e política industrial, como apontado por Chang (2004) e Egelhoff et al (2002), não é uma exclusividade de países subdesenvolvidos ou em desenvolvimento, esse elo é pronunciado também nos países desenvolvidos como no caso de Canadá, Irlanda e Reino Unido que usaram o IDE como ferramenta de política industrial.

Desse modo, no cenário recente de competição mundial por investimentos direto estrangeiro (IDE) em P\&D, as políticas nacionais destinadas a atrair esses investimentos não podem ser reduzidas a incentivos fiscais. Eles devem consistir de uma ampla gama de medidas capazes de criar um ambiente nacional adequado - em termos de instituições, infraestrutura, economia e educação - para atrair e promover esses investimentos (ZANATTA e QUEIROZ, 2007).

\section{METODOLOGIA}

O artigo apresenta uma análise bibliométrica com a finalidade de identificar uma amostra da produção científica sobre o tema em questão. De acordo com Soares et al. (2016), a pesquisa bibliométrica auxilia na identificação de tendências de crescimento do conhecimento em determinada disciplina, dispersão e obsolescências de campos científicos, autores e instituições mais produtivos, e periódicos mais utilizados na divulgação de pesquisas em determinada área do conhecimento.

Para realização deste estudo, adotou-se a seguinte sistemática de análise: na primeira etapa foram selecionadas cinco palavras chave alinhadas as temáticas de Política Industrial, Investimento Direto Externo e Transbordamentos de Produtividade: Industrial Policy; Foreign Investment Direct; Countries Developing; Spillover; Productivity. Desse modo, utilizou-se a plataforma Web of Science para coleta dos dados.

$\mathrm{Na}$ segunda etapa foram realizados os filtros de pesquisa. O levantamento de dados foi realizado em junho de 2018 e considerou as palavras chave definidas. Optou-se por filtrar a pesquisa pelos seguintes critérios: data de 
publicação (intervalo de 1980-2018), tipo de material (artigo), periódicos (revisados por pares) e descritores indicados em título, resumo ou palavras chave, totalizando 54 resultados.

A partir dos resultados, iniciou-se a terceira etapa de análise. Gerou-se a partir da plataforma Web of Science um arquivo com a extensão (.txt), que foi utilizado como entrada para análise no software CiteSpace. De acordo com Chen (2006), o CiteSpace permite detectar e visualizar as tendências da temática pesquisada a partir do estabelecimento de redes de trabalho que envolve autores, periódicos e séries temporais.

\section{ANÁLISE DOS RESULTADOS}

A partir da análise de redes autorais, percebe-se a frequência e relação entre os pesquisadores da área. Na Figura 1 são realizadas as análises de rede de autoria e coautoria.

Observa-se que nas áreas periféricas da figura encontram-se os autores que possuem publicações com menor número de parcerias e, caminhando para o centro, identifica-se autores que desenvolvem parcerias em suas publicações e pesquisas sobre o tema. No caso específico destaca-se Rene Belderbos com uma frequência de 27 citações em que trabalha como autor ou coautor. Outros autores se destacam como Javorcik, Blomstrom, Cohen. O tamanho dos nós identifica a quantidade de trabalhos de um pesquisador. Quanto maior o nó, maior a quantidade de trabalhos. A cor simboliza a quantidade de citações e, quanto mais intensa, maior a quantidade de citações.

Ao considerar a rede de colaboração por países, percebe-se que Estados Unidos, China e Inglaterra possuem a maioria dos pesquisadores que investigam as temáticas relacionadas à Política Industrial, Investimento Direto Externo e os Transbordamentos de Produtividade. Mesmo que a maioria dos estudos dessa linha abordem a implicação do investimento estrangeiro direto em países subdesenvolvidos ou em desenvolvimento, observa-se que existem poucas conexões entre pesquisadores dessas nacionalidades. Contudo, deve-se considerar a importante participação de pesquisadores chineses e indianos em alguns desses estudos.

Figura 1 - Redes de autoria e coautoria

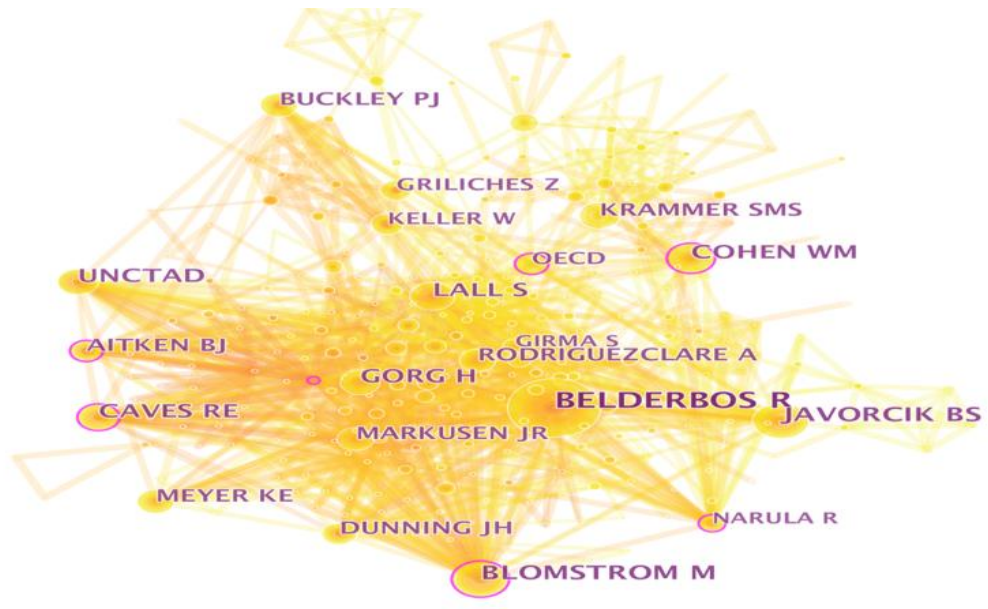

Fonte: elaborado pelos autores. 
Figura 2 -Redes de colaboração entre países

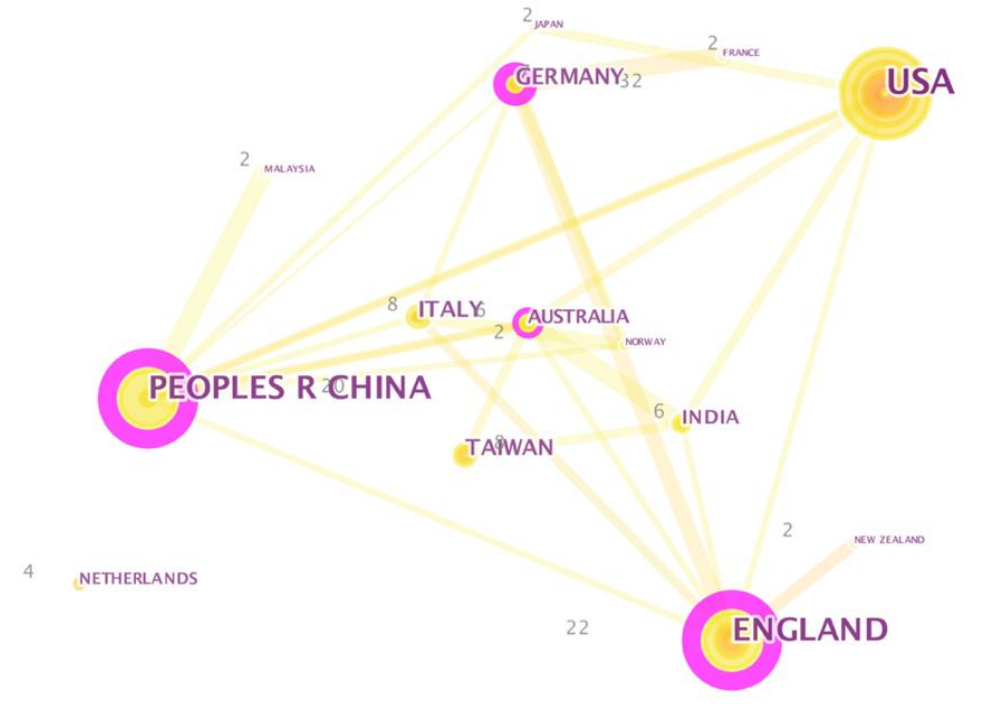

Fonte: elaborado pelos autores

Merece destacar nessa análise de redes que o termo mais citado é "foreign investment direct". A partir da conexão com esse termo surgem relevantes temáticas relacionadas como (i) países em desenvolvimento: são países que possuem um padrão de vida entre baixo e médio e com uma base industrial em desenvolvimento; (ii) crescimento econômico: refere-se ao aumento da capacidade produtiva da economia a partir da medição do valor dos bens e serviços que o país produz num período; (iii) pesquisa e desenvolvimento: atividades orientadas para a ciência e tecnologia que produzam pesquisas e estudos para o desenvolvimento econômico e social de um país; (iv) transbordamento (spillover): empresas de países anfitriões podem se apropriar da melhoria da produtividade e outros benefícios das empresas de propriedade estrangeira; (v) transferência de tecnologia: transferência de conhecimento cientifico ou tecnológico em combinação com fatores de produção e (vi) empresas multinacionais: empresas que possuem matriz num país e possuem atuação em diversos países. Para realçar a China e sua importância na economia global, o país é citado como exemplo de universo de pesquisa para trabalhos na temática analisada.

\section{CONSIDERAÇÕES FINAIS}

O objetivo do artigo foi realizar uma revisão bibliométrica a respeito de um tema considerado relevante para avaliar as tendências de crescimento do conhecimento, obsolescências e dispersão de campos científicos.

A forma como os países em desenvolvimento pode utilizar das políticas industriais para atração de investimentos estrangeiros e geração de transbordamento de produtividade em suas economias locais é um tema de relativa relevância, haja vista que, com esses transbordamentos, pode-se melhorar os resultados referentes ao crescimento econômico e se dissipar também para o bem-estar da população.

O estudo bibliométrico a partir da amostra de 54 artigos publicados entre 1980 e 
2018, demonstrou que a rede de autores e coautores encontrada está de acordo com o referencial teórico apresentado, bem como as formulações teóricas principais. Rene Belderbos destaca-se com uma frequência de 27 citações em que trabalha como autor ou coautor. O pesquisador tem como principal foco de pesquisa o desenvolvimento econômico e estratégias de negócios internacionais de empresas multinacionais. Em um de seus artigos "Backward vertical linkages of foreign manufacturing affiliates: Evidence from Japanese multinationals", Belderbos, Capanelli e Fukao (2001) examinam os determinantes das ligações verticais a montante estabelecidas por empresas multinacionais em economias hospedeiras por meio de uma análise da proporção de conteúdo local de 272 afiliadas japonesas de manufatura de eletrônicos em 24 países. Os fatores do país anfitrião que promovem os vínculos verticais são a qualidade da infraestrutura e o tamanho do setor de fornecimento de componentes locais, enquanto as políticas comerciais restritivas têm um efeito prejudicial. As regulamentações de conteúdo local têm um impacto positivo, mas não estimulam a aquisição de fornecedores locais. Nesse sentido, cabe ressaltar a frequência do termo "foreign investment direct" ou investimento direto estrangeiro.

O artigo pode conter uma limitação considerando a opção dos pesquisadores em utilizar essa estratégia de filtro, o que pode impactar na frequência das redes de autoria e coautoria. Desse modo, sugere-se que futuras pesquisas possam expandir a análise proposta incorporando ou retirando os filtros utilizados nesse artigo.

Um ponto a se destacar é que mesmo em se tratando de pesquisas cujo campo de aplicação se dá em países em desenvolvimento, a rede de autores e países (com exceção da China) ainda se concentra nos países ricos. Isso indica uma necessidade de os pesquisadores de economias emergentes lançarem esforços para este tema de bastante relevância para atual conjuntura econômica, com vistas a auxiliar nos seus ganhos de produtividade. Nesse sentido, novas pesquisas podem explorar as redes de autoria e coautoria de publicações oriundas de países emergentes e estabelecer comparativos a partir da frequência de acordo com cada região. Outrossim, destacase que a pesquisa realizada é relevante para a academia, tendo em vista que os estudos bibliométricos produzem registros bibliográficos que auxiliam os pesquisadores na compreensão da produção científica de uma determinada área temática de conhecimento..

\section{REFERÊNCIAS}

BELDERBOS, Rene; CAPANNELLI, Giovanni; FUKAO, Kyoji. Backward vertical linkages of foreign manufacturing affiliates: Evidence from Japanese multinationals. World development, v. 29, n. 1, p. 189-208, 2001.

BUCKLEY, Peter J.; CLEGG, Jeremy; WANG, Chengqi. Is the relationship between inward FDI and spillover effects linear? An empirical examination of the case of China. In: Foreign direct investment, China and the world economy. Palgrave Macmillan, London, 2010. p. 192-215

CAVES, Richard E. Multinational firms, competition, and productivity in host-country 
markets. Economica, v. 41, n. 162, p. 176-193, 1974.

CHANG, Ha-Joon. Regulation of foreign investment in historical perspective. The European Journal of Development Research, v. 16, n. 3, p. 687-715, 2004.

CHANG, Ha-Joon. Kicking away the ladder: development strategy in historical perspective. Anthem Press, 2002.

CHEN, C. CiteSpace II: detecting and visualizing emerging trends and transient patterns in scientific literature. Journal of the American Society for Information Science and Technology, v. 57, n. 3, p. 359-377, 2006.

CORONEL, Daniel Arruda; AZEVEDO, André Filipe Zago de; CAMPOS, Antônio Carvalho. Industrial policy and economic development: a review of the contemporary debate. Revista de Economia Política, v. 34, n. 1, p. 103-119, 2014.

COSTA, Ionara; FILIPPOV, Sergey. Foreignowned subsidiaries: a neglected nexus between foreign direct investment, industrial and innovation policies. Science and Public Policy, v. 35, n. 6, p. 379-390, 2008.

DEVLIN, Robert; MOGUILLANSKY, Graciela. What's new in the new industrial policy in Latin America?. In: The Industrial Policy Revolution I. Palgrave Macmillan, London, 2013. p. 276317.

DUNNING, John H. An overview of relations with national governments. New Political Economy, v. 3, n. 2, p. 280-284, 1998.

EGELHOFF, William G.; GORMAN, Liam; MCCORMICK, Stephen. How FDI characteristics influence subsidiary trade patterns: the case of Ireland. MIR:

Management International Review, p. 203230, 2000.

GÖRG, Holger; GREENAWAY, David. Much ado about nothing? Do domestic firms really benefit from foreign direct investment?. The World Bank Research Observer, v. 19, n. 2, p. 171-197, 2004.

LAZZARINI, Sergio G. Strategizing by the government: Can industrial policy create firm- level competitive advantage?. Strategic Management Journal, v. 36, n. 1, p. 97-112, 2015.

NARULA, R.; DUNNING, J. H. Industrial Development, Globalization and Multinational Enterprises: New Realities for Developing Countries. Oxford Development Studies, v. 28, n. 2, p. 141-167, 2000/06/01 2000.

NARULA, Rajneesh; DRIFFIELD, Nigel. Does FDI cause development? The ambiguity of the evidence and why it matters. The European Journal of Development Research, v. 24, n. 1, p. 1-7, 2012.

PACK, Howard; SAGGI, Kamal. Is there a case for industrial policy? A critical survey. The World Bank Research Observer, v. 21, n. 2, p. 267-297, 2006.

PRIMI, Annalisa; PERES NÚÑEZ, Wilson. Theory and practice of industrial policy: evidence from the Latin American experience. eclac, 2009.

RODRIK, Dani. Industrial policy for the twentyfirst century. CEPR Discussion paper n. 4767, Centre for Economic Policy Research, London, UK, 2004.

RUGMAN, Alan M.; VERBEKE, Alain. Multinational enterprises and public policy. In: International Business. Palgrave Macmillan, London, 2000. p. 21-43.

SOARES, Patrícia Bourguignon et al. Análise bibliométrica da produção científica brasileira sobre Tecnologia de Construção e Edificações na base de dados Web of Science. Ambiente Construído, v. 16, n. 1, p. 175-185, 2016.

STOPFORD, John M. The growing interdependence between transnational corporations and governments. Transnational Corporations, v. 3, n. 1, p. 53-76, 1994.

SUZIGAN, Wilson; FURTADO, João. Instituições e políticas industriais e tecnológicas: reflexões a partir da experiência brasileira. Estudos Econômicos (São Paulo), v. 40, n. 1, p. 7-41, 2010. 
VERNON, Raymond. In the hurricane's eye: the Troubled Prospects of Multinational

Enterprises. Harvard University Press, 1998.

ZANATTA, M.; QUEIROZ, S. The role of national policies on the attraction and promotion of MNEs' R\&D activities in developing countries. International Review of Applied

Economics, v. 21, n. 3, p. 419-435, 2007.

\section{Rafaela Maiara Caetano}

Doutoranda em Administração, UFLA. Mestre em Economia Aplicada pelo Programa de Pós-Graduação em Economia do Instituto de Economia da Universidade Federal de Uberlândia. Graduada em Ciências Econômicas pela Universidade Federal de São João del Rei. Faz parte do Grupo de Estudos em Economia Industrial e Negócios Internacionais (GEINI) da UFLA. E-mail: rafa.caet.94@gmail.com

\section{Sheldon William Silva}

Doutorando em Administração pela UFLA. Mestre em Administração pela FPL e graduação em Administração com Habilitação em Comércio Exterior pela UEMG. Professor no Centro Universitário do Sul de Minas. Faz parte do Grupo de Estudos em Economia Industrial e Negócios Internacionais (GEINI) da UFLA.

E-mail: sheldonwilliamsilva@gmail.com

\section{Dany Flavio Tonelli}

Docente do Departamento de Administração e Economia da UFLA. Possui doutorado em Organizações, Mudanças e Estratégia pelo Programa de Pós-Graduação em Administração da Universidade Federal de Lavras (PPGA/UFLA). É líder do grupo do grupo de pesquisa e vice coordenador do NIESP - Núcleo de Estudos em Inovação, Empreendedorismo e Setor Público.

E-mail: danytonelli@dae.ufla.br

\section{Cristina Lelis Leal Calegario}

Docente do Departamento de Administração e Economia da UFLA. Possui Doutorado em Agricultural and Applied Economics - University of Georgia (2005) e Estágio Sênior no Departamento de Management \& Global Business na Rutgers University, Newark, NJ (2015-2016).

Coordenadora por parte da UFLA do Consórcio em Educação Superior CAPES/FIPSE, desde 2008 com a UFRA e as Universidades americanas, North Michigan University e West Illinois University.

E-mail: ccalegario@dae.ufla.br
UNCTAD. World Investment Report 2018: Investment and new industrial policy. New York and Geneva: United Nations, 2018.

UNIDO, Structural Change. Poverty Reduction And Industrial Policy In The BRICS. UN

Publication, Vienna, 2012.

\author{
Gideon Carvalho de Benedicto \\ Docente do Departamento de Administração e Economia \\ da UFLA. Possui graduação em Ciências Contábeis pela \\ Faculdade de Ciências e Letras de Campo Mourão (1983); \\ Mestrado em Ciências Contábeis e Atuariais pela Pontifícia \\ Universidade Católica de São Paulo (1992); Doutorado em \\ Controladoria e Contabilidade pela USP (1997). E-mail: \\ gideon.benedicto@dae.ufla.br
}

\begin{tabular}{|c|c|}
\hline \multirow{2}{*}{$\begin{array}{l}\text { UNCTAD. World Investment Report 2018: } \\
\text { Investment and new industrial policy. New York } \\
\text { and Geneva: United Nations, } 2018 .\end{array}$} & \\
\hline & $\begin{array}{l}\text { Gideon Carvalho de Benedicto } \\
\text { Docente do Departamento de Administração e Economia } \\
\text { da UFLA. Possui graduação em Ciências Contábeis pela }\end{array}$ \\
\hline UNIDO, Structural Change. Poverty Reduction & $\begin{array}{l}\text { Faculdade de Ciências e Letras de Campo Mourão (1983); } \\
\text { Mestrado em Ciências Contábeis e Atuariais pela Pontifícia }\end{array}$ \\
\hline And Industrial Policy In The BRICS. UN & Universidade Católica de São Paulo (1992); Doutorado em \\
\hline Publication, Vienna, 2012. & $\begin{array}{l}\text { Controladoria e Contabilidade pela USP (1997). E-mail: } \\
\text { gideon.benedicto@dae.ufla.br }\end{array}$ \\
\hline
\end{tabular}

